$\xi=-$ 圈

\title{
An Automated Framework for Brain Tumour Class Detection
}

\author{
B. Nandan $^{1} *$, Kunjam Nageswara Rao ${ }^{2}$ \\ ${ }^{1}$ Research Scholar, Department of CS\&SE, AUCE(A), Andhra University, Visakhapatnam, AP, India \\ ${ }^{2}$ Associate Professor, Department of CS\&SE, AUCE(A), Andhra University, Visakhapatnam, AP, India \\ Corresponding author E-mail: b.nandan@gmail.com ${ }^{1 *}$, kunjamnag@gmail.com ${ }^{2}$
}

\begin{abstract}
With the significant growth in medical imaging techniques and the demand for better processing of medical information, the mandate of automation in disease detection is also increasing. In the modern time, the nature of the diseases has also changed. The highly mortal diseases are becoming difficult to detect due to the high involvements of medical individual and high dependency of human knowledges. The human knowledge is prone to error and often criticized for longer time delay for processing information in disease detections. Thus, the demand from the modern computing and implementation based computational algorithms are to automate the medical disease detection processes with greater accuracy. One such disease with superior mortal rate is brain tumours or cancerous growth in the brain tissues The regular medical practice approaches have demonstrated the challenges in detection of the tumours and more so the nature of the tumours. Ill detection of the tumour type or the shape of the tumour or the size of the tumours can lead to life threats. Thus, the need for automation in detection is the most expected form of replacements in place of manual diagnosis. Another challenge is the available data formats for such disease reports. The available reports for brain tumour are only in the form of magnetic resonance images or MR Images. The MR Images can cause higher obstacles for further processing as due to the capture process of the patient data. Often, it is observed that the noise present in the MR images makes the processing vulnerable in accuracy. A number of parallel research outcomes have demonstrated significant outcomes of detection of available tumours in the human brain using segmentation methods. Nonetheless, all parallel attempts are criticized for not able to model the growth or the nature of the tumours presents in the human brain. Thus, this work proposes a novel automated framework for detection of tumour types by deploying progressive segmentation and model the growth stages based on features. The parallel outcomes have outrun on detection accuracy due to the use of standard segmentation methods, which is designed for generic image processing and bound not to match the specificity of medical image processing. Thus, this work introduces a novel segmentation method, which is progressive in nature for higher accuracy. This work also outcomes into an automated feature extraction model for brain tumours. The major contribution of the work is to determine the nature of tumour and a sustainable prediction model for tumour stages inside the human brain. The work demonstrates high accuracy for correct detection and prediction of the patient's life threats in in real time order to take timely medication for making the precious human life more precious.
\end{abstract}

Keywords: Progressive Segmentation; Modelling Tumour Features; Tumour-Classifications \& Detections; MR Image Analysis; Image Based Modelling.

\section{Introduction}

The generic physiology of human body makes the brain as core of the nervous system. The adult brain size of the human body ranges between 1.3 to $1.4 \mathrm{Kgs}$. As per the most recent human brain imaging by K. L. Bigos et al. [1] demonstrates that the brain is comprising of soft tissues, neurons and glial cells. Though study of the human brain is still considered to be a challenge due to the complex structure, the researchers like S. Herculano-Houzel et al. [2] have made significant progress in estimating the number of cells or like the report from Brain Facts and Answers [3] estimated the number of connections in human brain. It is regardless to mention that, any damage or any disruption in the human brain cell can heavily damage the functions of the brain, nervous systems and for overall body. Thus, a huge number of researches are ongoing in terms of detection and for preventive cares of the human brain cells.

The major disruption of the brain functions can be observed in the presence of tumours in brain cells. These tumours can be highly aggressive in terms of growths over time. Thus, based on the aggressiveness of the cell division patterns or cell growths, the tumours can be classified in two classes as with a reduced pace growth or noncancerous growth can be identified as benign and with a high pace of growth or cancerous growth can be identified as malignant tumours. It is notable that, the damages from the brain tumours are usually not life threating rather can cause disfunctions in the whole body as reported in the study by L. M. De Angelis et al. [4].

The commonly found malignant brain tumours are called glioma. The glioma generates from the brain cell called glial, which is responsible for growth of the brain. The glioma can cause $80 \%$ of the situations of the malignant brain tumours as found in the study by M. L. Goodenberger et al. [5]. This analysis was made possible again with brain imaging techniques deployed by J. Meng et al. [6]. This study made the functioning of human brain much clear for further analysis and malfunctioning detection [Fig - 1].

Also, the gliomas can be further classified based on several characteristics and severity. The report published by World Health Organization have made some clear distinguishes, which is further reported by D. N. Louis et al. [7], for classifying the tumours as grade - I to grade - IV, where grade - I and II can be considered as low grade and grade - III and IV can be considered as high grades. The possibilities of transforming low grade to high grade is also significant as $60 \%$ of low grade gliomas are seen to get 
converted into a high grade as shown in the surveys by E. B. Claus et al. [8] and K. A. Jaeckle et al. [9].
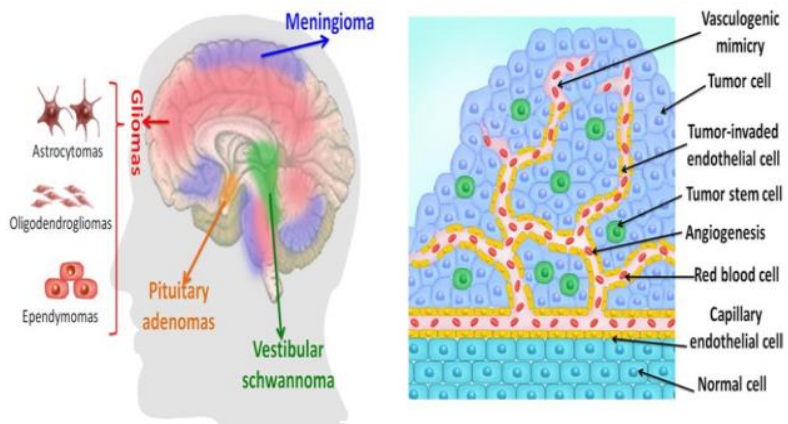

Fig. 1: Anatomy of Human Brain.

The detection of the brain cell functionalities or presence of any tumours or growth and location of tumours in the brain cell can be identified using magnetic resonance imaging or MRI methods. The process is generally occurring by deploying a specific contrast again called Gadolinium. The MR images based on specific characteristics of detecting features of brain cells or brain functioning can be classified in two four major groups as T1 analysis, T1 Gadolinium analysis, T2 analysis and finally FLAIR analysis. The graphical representations of the image types for these analyses can be easily distensible [Fig - 2]

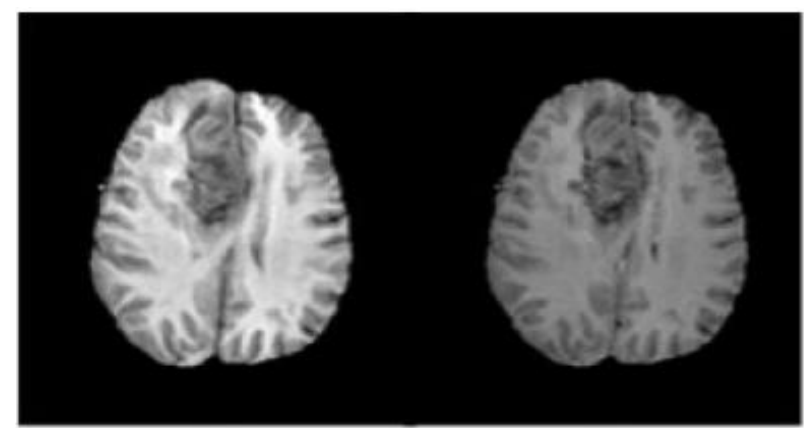

(a)

(b)

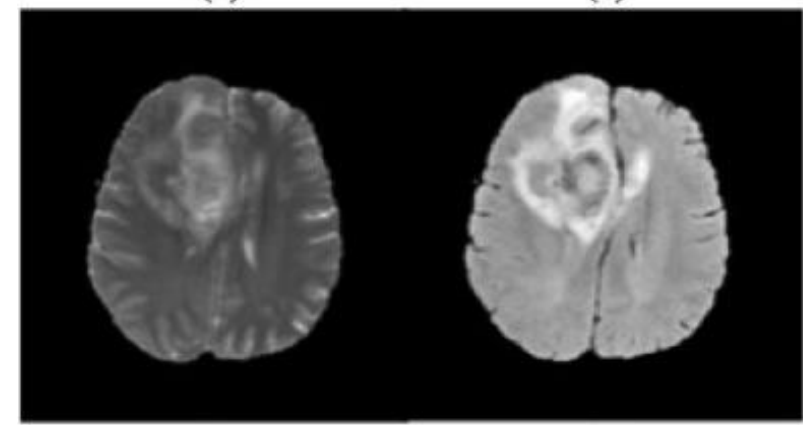

(c)

(d)

Fig. 2: Visual Differences of MR Image Types (A) TI, (B) T1 - Gadolinium, (C) T2 and (D) FLAIR.

The first three types are based on expansion of the brain cells during the functioning of brains and the last type is based on the recovery phases from the expansions.

The research on analysing and detecting brain tumours can be observed from a decade. The main aim for the studies, which are already present, are on the detection and modelling of tumour characteristics. The significant outcomes from these works are widely accepted and at the same point of time highly criticized as well due to the fact that these methods do not deploy the modern accurate methods for detection of tumour cells. Thus, the outcomes from these researches demands improvements. The recent review by J. C. L. Alfonso et al. [10] clearly shows the limitations in terms of accuracy for the existing methods. This review also includes the cross domain works by H. M. Byrne et al. [11] on cancerous growth detection, H. L. P. Harpold et al. [12] on mathematical modelling of the information available, work by $\mathrm{H}$. Hatzikirou et al. [13], work of A. H. Juffer et al. [14] on computational benefits on modelling growth, Y. Kam et al. [15] on applications of modelling and benefits, the work of N. L. Martirosyan et al. [16] on feature extractions on mathematical modelling, the application by N. Meghdadi et al. [17] based on medical imaging methods, A. Roniotis et al. [18] on diffusion method for growth modelling, the predictive framework by S. Sanga et al. [19], simulating the cell properties on nonliving situations by $\mathrm{Z}$. Wang et al. [20], on silico model by L. B. Edelman et al. [21] and finally the notable work by P. M. Altrock et al. [22] on cancer for human body cells.

Henceforth it is the demand from the recent research to include the advance imaging and mathematical models in order to improve the modelling mechanisms. This work deploys a progressive segmentation technique and further extraction of the features for tumour class detection with a high accuracy.

The rest of the work is organized such as in the Section - II, the recent outcomes from the parallel researches are elaborated, the Section - III defines the benefits of MR Imaging and classifications of the MR image types, in the Section - IV, the proposed matrix with tumour features is elaborated, in the Section $-\mathrm{V}$, the novel segmentation method is elaborated, in the Section - VI, the feature or attribute extraction algorithm is furnished, in the Section - VII, the rule based classification of tumour method is framed, in the Section - VIII, the complete working flow of the proposed framework is demonstrated, in the Section - IX, the obtained results from the framework in parts are explained and discussed, in order to demonstrate the advantages of the proposed framework in this work, the comparative analysis is done in the Section $-\mathrm{X}$ and the work presents the final conclusion in the Section - XI.

\section{Outcome of the Parallel Researcher}

The detection of tumours and cancerous behaviours in human brain cells are one of the most popular area of research over a decade now. A good number of research attempts can be considered in order to understand the progress made by various researchers. Majorly, these attempts can be classified in three major categories as vivo, vitro and silico as classified by E. Konukoglu et al. [23]. Also, the types of analysis can be classified in three categories as well namely continuous, discrete and hybrid based on the growth patterns of the tumours as clearly stated by $\mathrm{Z}$. Wang et al. [24] in their work.

The framework proposed in this work highly relates to silico category and hybrid analysis for modelling and detection categories respectively.

The notable work by M. A. J. Chaplain et al. [25] made the analysis of growth phases much clear based on the growth pattern analysis for hybrid tumour types. The contributions of T. Roose et al. [26] also cannot be ignored for presenting a much simpler mathematical model for growth measurements. The benefits of mathematical modelling based on features is significant and makes any study high accurate as demonstrated by R. P. Araujo et al. [27]. Also, the general level-set methods are quite accurate for the simulation of tumour growths as showcased by C. S. Hogea et al. [28]. The hybrid models are comparatively more difficult for analysis and modelling or prediction as proven by J. D. Humphrey et al. [29].

Henceforth, this work applies the proposed methods to reduce the complexity and increase the accuracy compared to the existing methods.

The accuracy and the features highly depend on the types of the MR images. Thus, it is highly suggestable that, for any study using brain imagining techniques, the types of the MR images must be analysed.

The next section of this work analyses the MR image types and the associated benefits. 


\section{MR Image Classifications}

The MR images, as highlighted in the previous sections of this work, can be classified in four major classes. In this section of the work, the benefits from each type is elaborated.

Firstly, the Spin - Lattice unwinding time or T1 variation picture is indicated as rot steady for the recuperation time for the turn charge. The visual representations can be observed here [Fig - 3]. The sample is collected from BRATs 2017 dataset.

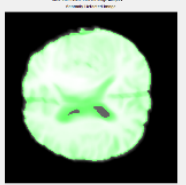

Patient -1

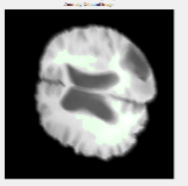

Patient -5

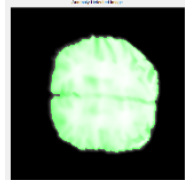

Patient -2

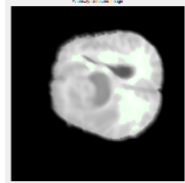

Patient -6

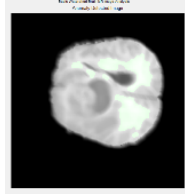

Patient -9

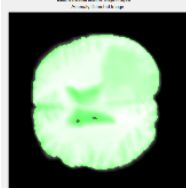

Patient -3

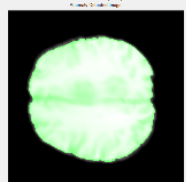

Patient -7

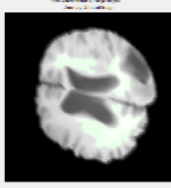

Patient -10

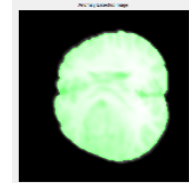

Patient -4

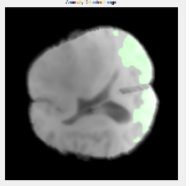

Patient -8
Fig. 3: Visual Differences of MR Image Types - T1.

Secondly, the transverse turn to turn unwinding time $\mathrm{T} 2$ is the rot consistent for the recuperation time for the turn polarization, where the charge vector rot towards the balance [Fig - 4]. The sample is collected from BRATs 2017 dataset.

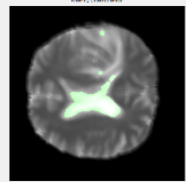

Patient -1

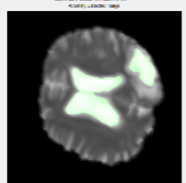

Patient -5

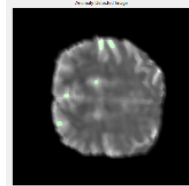

Patient -2

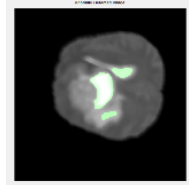

Patient -6

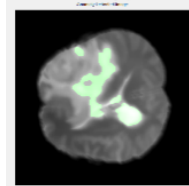

Patient -9

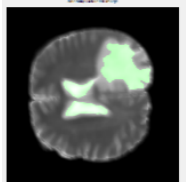

Patient -3

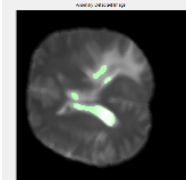

Patient -7

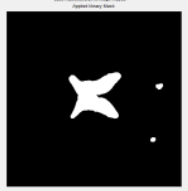

Patient -10

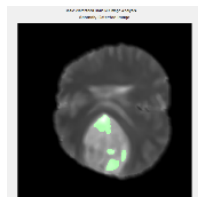

Patient -4

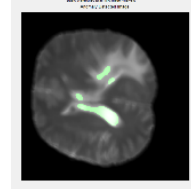

Patient -8
Fig. 4: Visual Differences of MR Image Types - T2.

Third, the T1C is the concentrated Spin - Lattice unwinding imaging which is like $\mathrm{T} 1$ pictures with the higher grouping of the attractive reverberation extents. The subtle elements of the $\mathrm{T} 1$ picture is as of now been exhibited in the past sub area [Fig - 5]. The sample is collected from BRATs 2017 dataset.

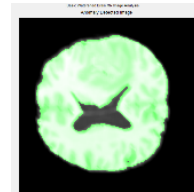

Patient -

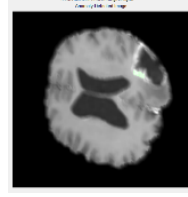

Patient -5

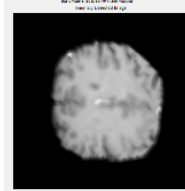

Patient -2

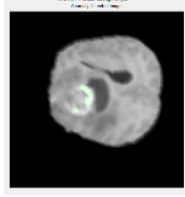

Patient -6

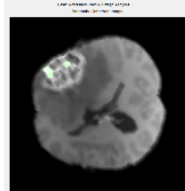

Patient -9

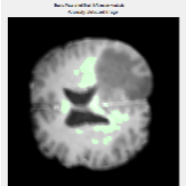

Patient -3

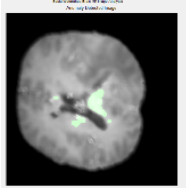

Patient -7

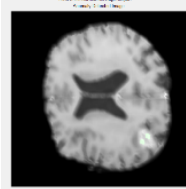

Patient -10

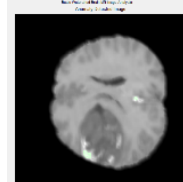

Patient -4

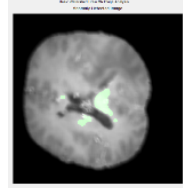

Patient -8
Fig. 5: Visual Differences of MR Image Types - T1C or Gadolinium.

Finally, the FLAIR or Fluid Attenuated Inversion Recovery is additionally a sort of picture produced by attractive reverberation to imagine the beat succession. The beat grouping can be gotten by applying Fourier change on any attractive unwinding yields and applying the rot. Henceforth, the result of FLAIR is likewise comparative as $\mathrm{T} 1, \mathrm{~T} 2$ and $\mathrm{T} 1 \mathrm{C}$. By the by, the significance of FLAIR can't be overlooked for the areas where the organ liquid is high and hindrances the best perception of attractive unwinding like the cerebrospinal liquid in the event of mind MR imaging [Fig -6]. The sample is collected from BRATs 2017 dataset.

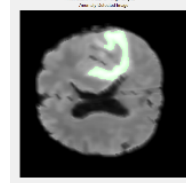

Patient -1

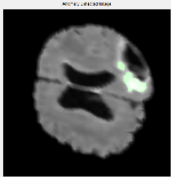

Patient -5

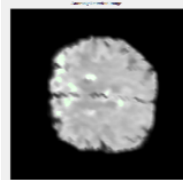

Patient -2

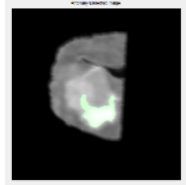

Patient -6

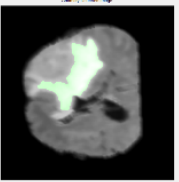

Patient -9

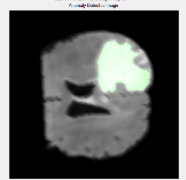

Patient -3

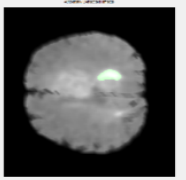

Patient -7

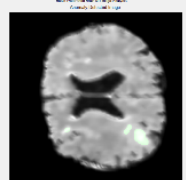

Patient -10

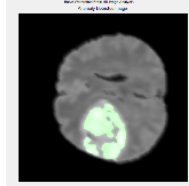

Patient -4

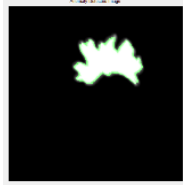

Patient -8

Fig. 6: Visual Differences of MR Image types - FLAIR.

Among the assortment of imaging modalities, Magnetic Resonance Imaging (MRI) indicates most points of interest of cerebrum and is the most well-known test for determination of mind tumours. X-ray contains T1-weighted MRI (T1w), T1-weighted MRI with differentiate improvement (T1wc), T2-weighted MRI (T2w), Proton Density-Weighted MRI (PDw), Fluid-Attenuated Inversion Recovery (FLAIR), et cetera. Not at all like Computed Tomography (CT) picture, have MRI pictures from various sorts of machines had diverse dark scale esteems.

Henceforth, from above knowledge, it is understandable that the features to be extracted for any further modelling must comply with all the imaging types of MR. Thus, in the next section of this work the proposed feature list is explained. 


\section{Proposed Brain Tumour Detection Parame- ters Matric}

Characterization or modelling or detection of the brain tumour needs a specific set of matric. This section of the work defines the matric used in this proposed framework. The matrices used in this kind of works, must comply with the underlying imaging techniques. Thus, this proposed matric considers the list of features which can be extracted from any MR image types.

The details of the proposed feature list or the matric for tumour analysis is elaborated here [Table - I].

Table- I: Tumour Detection Parameter Matric

\begin{tabular}{|c|c|c|}
\hline $\begin{array}{l}\text { Name of the } \\
\text { attribute }\end{array}$ & Description & $\begin{array}{l}\text { Value Range for Classifi- } \\
\text { cation }\end{array}$ \\
\hline Species & $\begin{array}{l}\text { The type of the tumour } \\
\text { based on the growth rate }\end{array}$ & $\begin{array}{l}\text { Three Possible values: } \\
\text { A. BENIGN } \\
\text { B. MALIGNANT } \\
\text { C. HYBRID }\end{array}$ \\
\hline Size & $\begin{array}{l}\text { The size of the tumour in } \\
\mathrm{mm} \text {. }\end{array}$ & $\begin{array}{l}\text { Min size } 0.0020 \\
\text { Max size } 0.0065\end{array}$ \\
\hline Entropy & $\begin{array}{l}\text { Defines the state of the } \\
\text { brain without any measur- } \\
\text { ing scale }\end{array}$ & $\begin{array}{l}\text { The higher entropy de- } \\
\text { termines higher level of } \\
\text { disorders }\end{array}$ \\
\hline RMS & $\begin{array}{l}\text { The rostral migratory } \\
\text { stream or RMS is a special- } \\
\text { ized migratory route found } \\
\text { in the brain denoting regu- } \\
\text { lar smelling capabilities of } \\
\text { brain. }\end{array}$ & $\begin{array}{l}\text { Less denotes loss of } \\
\text { smelling capabilities or } \\
\text { effect on other sensory } \\
\text { functions. } \\
\text { Average range is } 0.09 \text { to } \\
0.08 \text {. }\end{array}$ \\
\hline Variance & Variance of brain volume & $\begin{array}{l}\text { Grouped in ages of } 3,9 \text {, } \\
12,35\end{array}$ \\
\hline Smoothness & $\begin{array}{l}\text { Smoothness of the brain } \\
\text { cell surface }\end{array}$ & $\begin{array}{l}\text { Low denotes presence of } \\
\text { Lissencephaly, caused by } \\
\text { tumorous cells }\end{array}$ \\
\hline Kurtosis & $\begin{array}{l}\text { Kurtosis defines the sharp- } \\
\text { ness of the brain signals } \\
\text { during MRI analysis }\end{array}$ & As high as better \\
\hline Skewness & $\begin{array}{l}\text { Skewness defines asym- } \\
\text { metry of the brain respon- } \\
\text { siveness }\end{array}$ & As low as better \\
\hline IDM & $\begin{array}{l}\text { IDM denotes the electrical } \\
\text { activities of the brain. }\end{array}$ & $\begin{array}{l}\text { The negative denotes less } \\
\text { delay in the brain signal } \\
\text { processing capabilities. }\end{array}$ \\
\hline Contrast & $\begin{array}{l}\text { The amount of contrast } \\
\text { agents used during the MRI } \\
\text { process }\end{array}$ & $\begin{array}{l}\text { Defers based on various } \\
\text { clinical practices }\end{array}$ \\
\hline Correlation & $\begin{array}{l}\text { The clinical correlation } \\
\text { defines the severity of } \\
\text { demand for further analy- } \\
\text { sis. }\end{array}$ & $\begin{array}{l}\text { As high as highly rec- } \\
\text { ommended for further } \\
\text { analysis }\end{array}$ \\
\hline Energy & $\begin{array}{l}\text { The energy generated in the } \\
\text { human brain due to electron } \\
\text { transmissions }\end{array}$ & $\begin{array}{l}\text { As high as effective. } \\
\text { Average is } 0.7 \text { kilo calo- } \\
\text { ries }\end{array}$ \\
\hline Homogeneity & $\begin{array}{l}\text { The functional homogenei- } \\
\text { ty of the brain defines the } \\
\text { working pattern of the brain } \\
\text { functions }\end{array}$ & $\begin{array}{l}\text { As high as better. } \\
\text { Average is } 0.6 \text {. }\end{array}$ \\
\hline
\end{tabular}

Henceforth, these features can be extracted from various magnetic resonance imaging techniques and can be used for further analysis or modelling the nature of the tumours.

Nonetheless, the features can only be extracted from the MR images, if the segmentation of the brain tumour is correct. Thus, the next section of this work, elaborates on the proposed improved and specific segmentation algorithm for brain tumour detections.

\section{Proposed Novel Progressive Segmentation Algorithm}

The available segmentation methods used in the parallel research outcomes are not specifically designed for brain tumours or for brain imaging. The existing segmentation algorithms are made applicable for the brain images after applying specific pre- processing measures. It is regardless to mention, that as an effect of pre-process on any images, the image bound to loss some amount of information. In the space of regular image processing, the information loss is marginal as loss of few pixel values will certainly not underrate the results. Nonetheless, in case of medical imaging, loss of little pixel information can lead to highly inaccurate results.

Thus, this work presents a medical image specific segmentation algorithm.

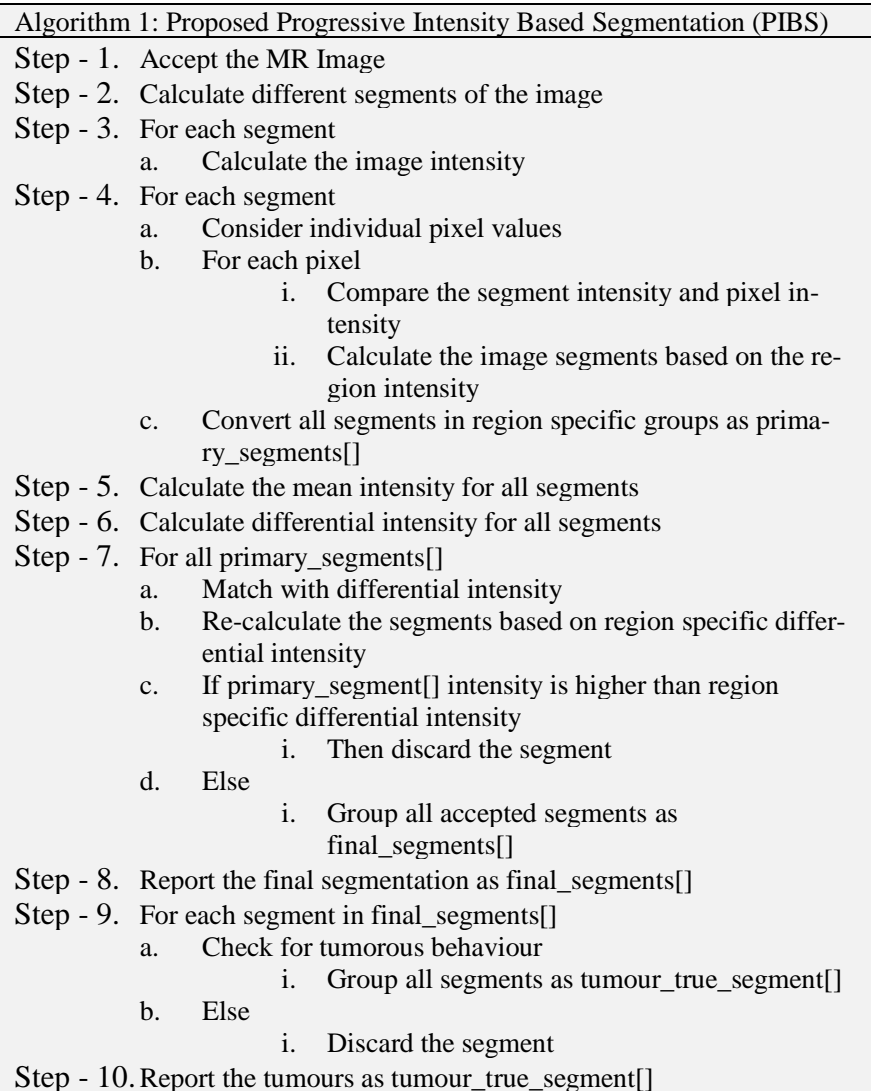

The algorithm flow is analysed graphically as well [Fig - 7]. 


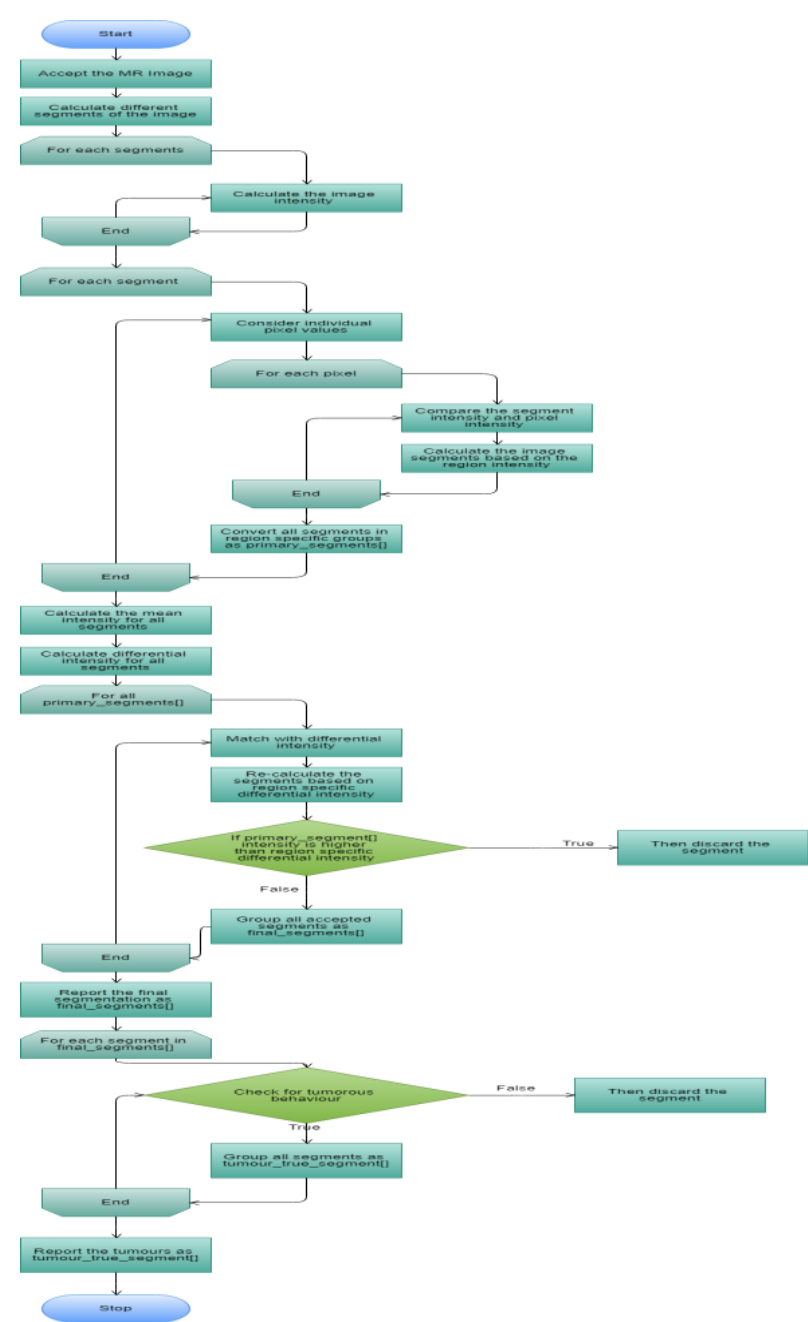

Fig. 7: PIBS Algorithm Flow.

Further, once the regions with the tumours are identified, the features or the parameter metric must be formulated. In the next section of the work, the feature extraction algorithm is elaborated.

\section{Proposed Tumour Attribute Extraction Algorithm}

The feature-based tumour detection and modelling methods are highly acceptable due to the fact that, the differentiation of the tumour classes can be easily identified based on feature metric After the detection of the segments on the MR Image with the presence of tumours, the specified segments can be supplied to the proposed feature extraction algorithm as demonstrated in this section of the work.

\begin{tabular}{ll}
\hline Algorithm - 2: Proposed Feature Extraction from MR Image (FEMRI) \\
\hline Step - 1. & Accept the segments with tumours \\
Step - 2. Apply curve fitting method on tumour regions \\
Step - 3. & For each shape \\
a. Calculate the area \\
b. Report each tumour size \\
Step - 4. Convert the digital MR image into analogue signal \\
a. Calculate the entropy of the signal \\
b. Report the entropy as final entropy of the brain \\
c. Calculate the sharpness of the signal \\
d. Report the entropy pattern as Homogeneity \\
e. Report the sharpness as brain Kurtosis \\
f. Calculate the Skewness of the signal \\
g. Report the Skewness as brain Skewness \\
h. Calculate the Energy of the signal \\
i. Report the Energy as brain Energy \\
Step - 5. Calculate the IDM for the Brain Signals \\
a. Report the IDM for brain \\
Step - 6. Calculate the density of the nervous system cells \\
\hline
\end{tabular}

\begin{tabular}{|c|c|}
\hline & $\begin{array}{ll}\text { a. } & \text { Report the density as RMS } \\
\text { b. } & \text { Report the Contrast }\end{array}$ \\
\hline Step - 7. & Calculate the total brain cell area \\
\hline & Apply curve fitting method on total area \\
\hline Step - 8. & $\begin{array}{l}\text { Calculate the area for each shape } \\
\text { a. Report the total area as variance of the brain }\end{array}$ \\
\hline Step - 9. & $\begin{array}{l}\text { Apply Edge-Detection method on the MR image } \\
\text { a. Calculate the variation of the edge }\end{array}$ \\
\hline & b. Report the variation of the edge pixels as smoothness \\
\hline Step - 10. & $\begin{array}{l}\text { Calculate Correlation with the final results } \\
\text { a. Report the Correlation }\end{array}$ \\
\hline
\end{tabular}

The algorithm flow is analysed graphically as well [Fig - 8].

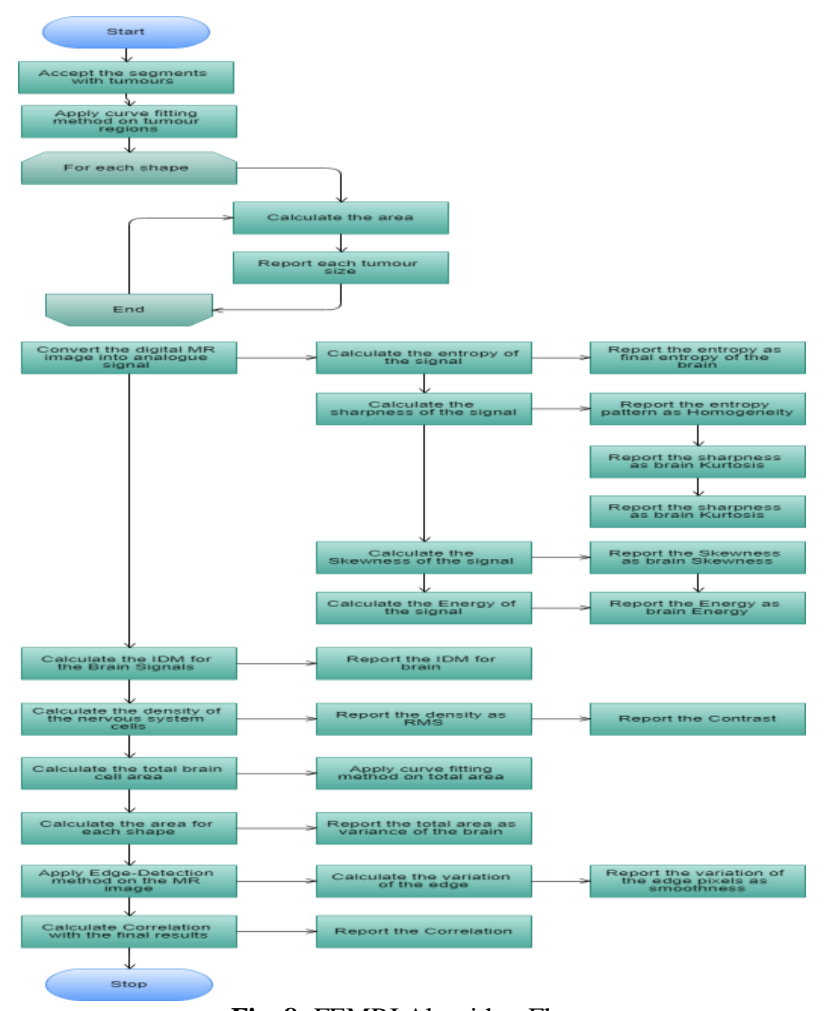

Fig. 8: FEMRI Algorithm Flow.

Once the feature sets are extracted for each tumour for each dataset images, the classification of the tumours can be applied. The proposed class detection algorithm is furnished in the next section.

\section{Proposed Tumour Class Detection Algorithm}

The purpose of the image segmentation is to make the segments applicable to feature extraction and the purpose of feature extraction is to make the rule engine for tumour classifications.

Henceforth, this section of the work elaborates on the rule-based algorithm for tumour class detection.

\begin{tabular}{ll}
\hline Algorithm - 3: Apriori Based Rule Engine for Tumour Class Detection \\
(ABRETCD)
\end{tabular}


The final analysis of the rule engine is visualized here [Fig - 9].

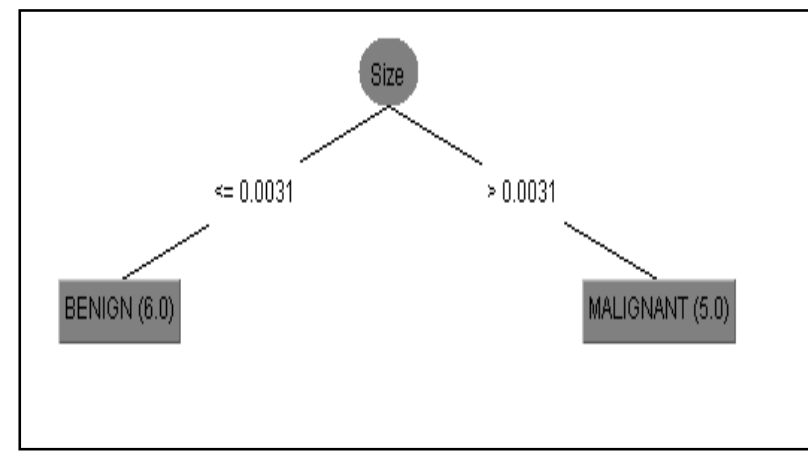

Fig. 9: Final Analysis of the Rule Engine Algorithm Flow.

In the next section of this work, the complete workflow of the proposed automated framework is elaborated.

\section{Proposed Framework}

The primary purpose of the proposed automated framework for detecting the class of the brain tumour. Nevertheless, the automated framework is also capable of reporting the size and entropy of the brain during the detection process as well. The [Fig-10] shows that, it accept the MR Images as input from dataset BRATS-2017 and apply the PIBS Algorithm for Segmentation, further output of the Segments are applied to FERMI Algorithm for feature extraction for all the eleven patients images further analysis. FERMI output is applied to ABRETCD Algorithm to determine the tumour classes as Benign or Malignant.

The complete flow of the proposed framework is enlisted here in this section of the work [Fig - 10].

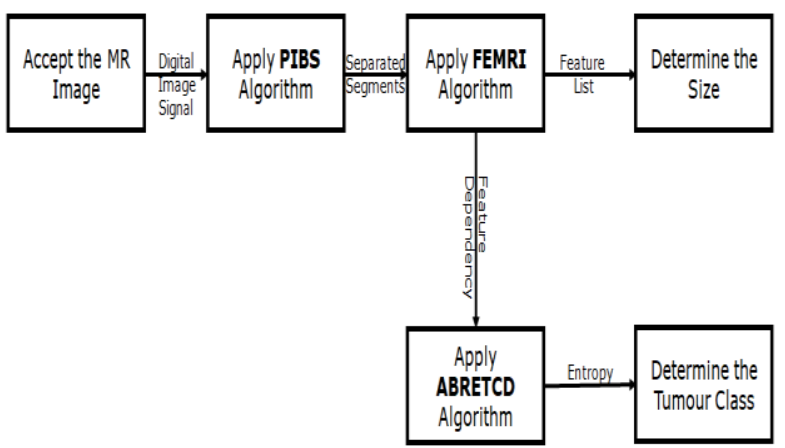

Fig. 10: Processing Flow of the Proposed Algorithm.

Henceforth, in the further section of this work, the obtained results from the proposed framework are discussed.

\section{Results and Discussions}

The results obtained from the proposed framework are highly satisfactory and are discussed in this section. The results are divided as per the framework components with Segmentation, Feature List extraction, Detection of tumour presence, Classification of the tumours, RBF kernel Accuracy, Linear kernel Accuracy, Polynomial kernel Accuracy and finally Quadratic kernel Accuracy.

a) segmentation Phase Result
Firstly, the segmentation results are analysed [Table - II]. The information of existing number of segments are available in the dataset for each patient records and further, the proposed progressive segmentation algorithm is applied on the same dataset to match the accuracy.

Table- II: Segmentation Accuracy

\begin{tabular}{cccc}
\hline Dataset & $\begin{array}{l}\text { Actual Number of } \\
\text { Segments with Tu- } \\
\text { mours }\end{array}$ & $\begin{array}{l}\text { Number of Segments } \\
\text { Detected with Tu- } \\
\text { mours }\end{array}$ & $\begin{array}{l}\text { Accuracy } \\
(\%)\end{array}$ \\
\hline Patient-1 & 1 & 1 & 100 \\
Patient-2 & 1 & 1 & 100 \\
Patient-3 & 1 & 1 & 100 \\
Patient-4 & 1 & 1 & 100 \\
Patient-5 & 2 & 2 & 100 \\
Patient-6 & 2 & 1 & 50 \\
Patient-7 & 1 & 1 & 100 \\
Patient-8 & 1 & 1 & 100 \\
Patient-9 & 1 & 1 & 100 \\
Patient-10 & 1 & 1 & 100 \\
Patient-11 & 1 & 1 & 100 \\
\hline
\end{tabular}

The results are analysed graphically as well [Fig - 11].

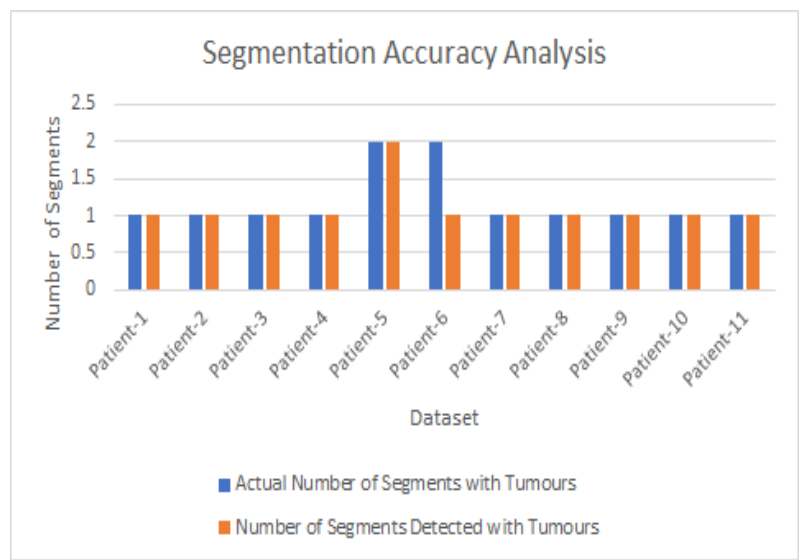

Fig. 11: Segmentation Accuracy Analysis.

It is natural to understand that the accuracy of the proposed progressive segmentation algorithm is very high and satisfactory.

b) Feature Extraction

Secondly, the feature extraction algorithm results are furnished here [Table - III]. The extracted features are as proposed in the previous section of this work. 
Table- III: Feature Extraction Accuracy

\begin{tabular}{|c|c|c|c|c|c|c|c|c|c|c|c|c|}
\hline Dataset & Size & Entropy & RMS & Variance & Smoothness & Kurtosis & Skewness & IDM & Contrast & Correlation & Energy & Homogeneity \\
\hline Patient-1 & 0.0031 & 3.1735 & 0.0898 & 0.008 & 0.9205 & 7.3282 & 0.469 & 0.0577 & 0.2088 & 0.199 & 0.7621 & 0.9352 \\
\hline Patient-2 & 0.0031 & 3.1735 & 0.0898 & 0.008 & 0.9205 & 7.3282 & 0.469 & 0.0577 & 0.2088 & 0.199 & 0.7621 & 0.9352 \\
\hline Patient-3 & 0.0024 & 3.2698 & 0.0898 & 0.0081 & 0.8974 & 7.9567 & 0.8862 & 0.4926 & 0.2717 & 0.0931 & 0.7686 & 0.9338 \\
\hline Patient-4 & 0.003 & 3.555 & 0.0898 & 0.008 & 0.9178 & 6.3661 & 0.6499 & 0.4729 & 0.2414 & 0.1065 & 0.744 & 0.9277 \\
\hline Patient-5 & 0.0025 & 3.3156 & 0.0898 & 0.0081 & 0.9032 & 6.232 & 0.3121 & 0.5631 & 0.2161 & 0.1382 & 0.7548 & 0.9325 \\
\hline Patient-6 & 0.0021 & 3.5182 & 0.0898 & 0.008 & 0.885 & 6.7672 & 0.4413 & 0.5462 & 0.225 & 0.0991 & 0.7691 & 0.9365 \\
\hline Patient-7 & 0.0063 & 3.2051 & 0.0898 & 0.008 & 0.9591 & 12.2408 & 1.1048 & 1.2156 & 0.3059 & 0.1421 & 0.7862 & 0.9379 \\
\hline Patient-8 & 0.0043 & 3.6004 & 0.0898 & 0.0081 & 0.9407 & 6.0137 & 0.5267 & 0.3801 & 0.2255 & 0.1345 & 0.7466 & 0.9299 \\
\hline Patient-9 & 0.0037 & 3.371 & 0.0898 & 0.0081 & 0.9314 & 7.3506 & 0.635 & 0.1378 & 0.2433 & 0.0933 & 0.7613 & 0.9329 \\
\hline Patient-10 & 0.0046 & 3.029 & 0.0898 & 0.0081 & 0.9453 & 13.1839 & 1.0085 & 0.2863 & 0.275 & 0.118 & 0.7688 & 0.9346 \\
\hline Patient-11 & 0.0042 & 3.5516 & 0.0898 & 0.008 & 0.9403 & 6.0614 & 0.5104 & 0.313 & 0.2314 & 0.1072 & 0.7418 & 0.9298 \\
\hline
\end{tabular}

The extracted features are visualized graphically as well [Fig - 12].

Table- IV: Feature Extraction Accuracy

\begin{tabular}{|c|c|c|c|c|c|c|c|c|c|c|c|c|}
\hline Dataset & Size & Entrony & RMS & Variance & & Kurtocic & & & & & & \\
\hline $\mathrm{T} 1$ & 1 & 1 & 1 & 1 & 0 & 1 & 1 & 1 & 1 & 1 & 1 & 1 \\
\hline $\mathrm{T} 1 \mathrm{C}$ & 1 & 1 & 1 & 0 & 1 & 1 & 1 & 1 & 1 & 1 & 1 & 1 \\
\hline Flair & 1 & 0 & 0 & 1 & 1 & 1 & 1 & 1 & 0 & 1 & 1 & 1 \\
\hline
\end{tabular}

Feature Extraction Analysis



Fig. 12: Feature Extraction Analysis.

Also, the given features must be extractable from all types of MR images. Thus, this work performs the extractability analysis as well [Table - IV].

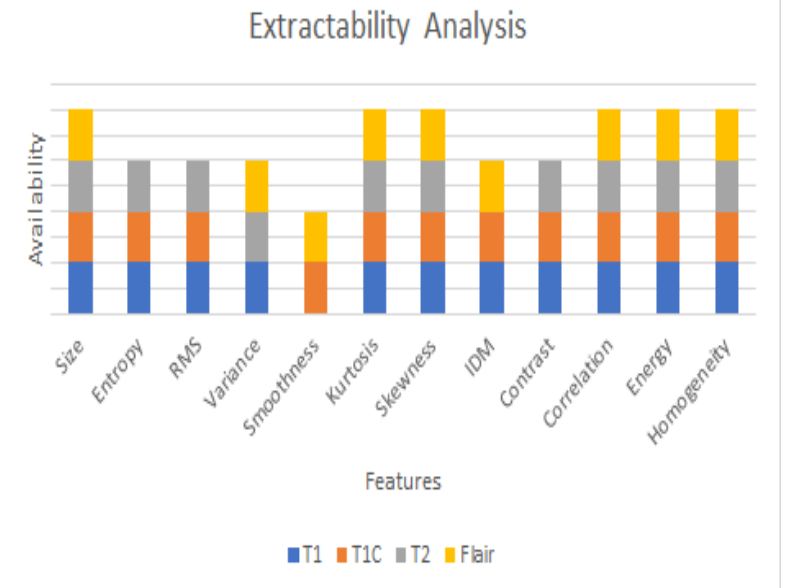

Fig. 13: Feature Extraction Analysis.

Henceforth, it is usual to understand that, the features proposed in this work can be extracted from all possible MR image types and are widely accepted.

c) Detection of Tumours

Third, the detection of the tumours or the availability of the tumour cells in the brain results are elaborated here [Table - V]. The True Positive rate (TP) or Success rate is 1, False Positive rate (FP) or Misclassification error is 0, Precision (Specificity) given as $(\mathrm{FP} / \mathrm{FP}+\mathrm{TN})$ is 1 , Recall (Sensitivity) given as $(\mathrm{TP} / \mathrm{TP}+\mathrm{FP})$ is 1 , F-Measure is 1 and ROC Area is 1 for Benign and Malignant is correctly classified in our proposed method as shown in [Table-V].

Table- V: Classification of Tumours

\begin{tabular}{|c|c|c|c|c|c|c|c|}
\hline & TP Rate & FP Rate & Precision & Recall & F-Measure & ROC Area & Class \\
\hline & 1 & 0 & 1 & 1 & 1 & 1 & BENIGN \\
\hline & 1 & 0 & 1 & 1 & 1 & 1 & MALIGNANT \\
\hline Weighted Avg. & 1 & 0 & 1 & 1 & 1 & 1 & \\
\hline
\end{tabular}

Thus, it can be realized that the proposed tumour detection algorithm can identify two available classes of tumours in the dataset.

d) Classification of Tumours

The major objective of this work is to classify the types of the tumours in the human brain cell. Based on the extracted features this work produces the classification and the results are compared with the dataset information [Table - VI].

Table VI: Classification Accuracy

\begin{tabular}{llll}
\hline Dataset & Actual Species & Detected Species & Accuracy $(\%)$ \\
\hline Patient-1 & BENIGN & BENIGN & 100 \\
\hline
\end{tabular}

\begin{tabular}{llll}
\hline Patient-2 & BENIGN & BENIGN & 100 \\
Patient-3 & BENIGN & BENIGN & 100 \\
Patient-4 & BENIGN & BENIGN & 100 \\
Patient-5 & BENIGN & BENIGN & 100 \\
Patient-6 & BENIGN & BENIGN & 100 \\
Patient-7 & MALIGNANT & MALIGNANT & 100 \\
Patient-8 & MALIGNANT & MALIGNANT & 100 \\
Patient-9 & MALIGNANT & MALIGNANT & 100 \\
Patient-10 & MALIGNANT & MALIGNANT & 100 \\
Patient-11 & MALIGNANT & MALIGNANT & 100 \\
\hline
\end{tabular}

The results are visualized graphically as well [14]. 


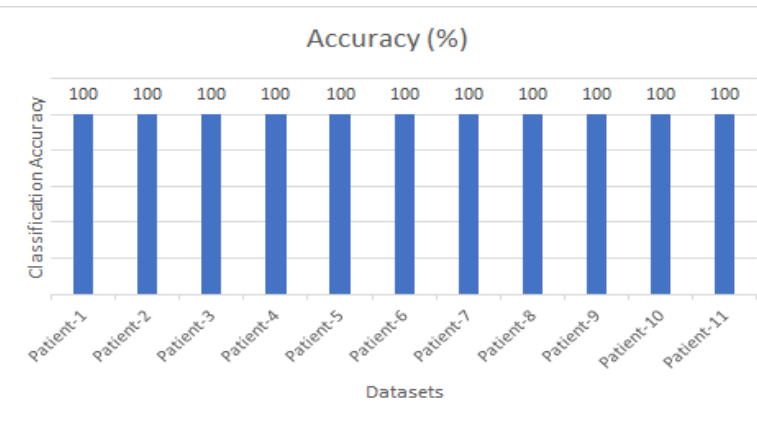

Fig. 14: Classification Accuracy Analysis.

The higher accuracy demonstrates improvements in the proposed method.

e) RBF Kernel Accuracy

A radial basis function (RBF) is a genuine esteemed capacity whose esteem depends just on the separation from the source, the standard is generally Euclidean separation, albeit other separation capacities are additionally conceivable. Entireties of outspread premise capacities are normally used to roughly given capacities. The RBF kernel on two samples $\mathrm{x}$ and $\mathrm{x}^{1}$ represented as feature vectors in some input space is $\mathrm{k}\left(\mathrm{x}, \mathrm{x}^{1}\right)=\exp \left(-\left\|\mathrm{x}-\mathrm{x}^{1}\right\|^{2} / 2 \sigma^{2}\right)$.

This section of the work, analyses the RBF accuracy of the proposed method for all the eleven patients from dataset is shown in [Table-VII].

Table-VII: RBF Kernel Accuracy

\begin{tabular}{lc}
\hline Dataset & Accuracy $(\%)$ \\
\hline Patient-1 & 70 \\
Patient-2 & 70 \\
Patient-3 & 80 \\
Patient-4 & 0 \\
Patient-5 & 70 \\
Patient-6 & 0 \\
Patient-7 & 0 \\
Patient-8 & 70 \\
Patient-9 & 70 \\
Patient-10 & 70 \\
Patient-11 & 0 \\
\hline
\end{tabular}

The graphical analysis of the accuracy is done at a further section of the work.

\section{f) Linear Kernel Accuracy}

The linear kernel has only one parameter, an additive constant. Any floating-point value is allowed for this parameter. For degree, $\mathrm{d}=1$, the linear kernel is defined as $\mathrm{K}(\mathrm{x}, \mathrm{y})=\left(\mathrm{x}^{\mathrm{T}} \mathrm{y}+\mathrm{c}\right)^{\mathrm{d}}$, where $\mathrm{x}$ and $\mathrm{y}$ are the vectors in the input space and $\mathrm{c}$ is a free parameter. Using Linear Kernel method for all the patients the accuracy percentages are obtained as shown in [Table-VIII]

This section of the work, analyses the Linear accuracy of the proposed method [Table - VIII].

Table VIII: Linear Kernel Accuracy

\begin{tabular}{ll} 
& Table VII: Linear Kernel Accuracy \\
\hline Dataset & Accuracy $(\%)$ \\
\hline Patient-1 & 80 \\
Patient-2 & 80 \\
Patient-3 & 90 \\
Patient-4 & 80 \\
Patient-5 & 80 \\
Patient-6 & 80 \\
Patient-7 & 90 \\
Patient-8 & 90 \\
Patient-9 & 90 \\
Patient-10 & 80 \\
Patient-11 & 90 \\
\hline
\end{tabular}

The graphical analysis of the accuracy is done at a further section of the work. g) Polynomial Kernel Accuracy

For degree, d, the Polynomial kernel is defined as $\mathrm{K}(\mathrm{x}, \mathrm{y})=\left(\mathrm{x}^{\mathrm{T}} \mathrm{y}+\mathrm{c}\right)^{\mathrm{d}}$, where $\mathrm{x}$ and $\mathrm{y}$ are the vectors in the input space and $\mathrm{c}$ is a free parameter. Using Polynomial Kernel method for all the patients the accuracy percentages are obtained as shown in [Table-IX]. In the event that the quantity of highlights is expansive, one should not have to outline to a higher dimensional space. That is, the nonlinear mapping does not enhance the execution. Nevertheless, for the fact of confirmation, the polynomial analysis is also carried out in this work [Table - IX].

Table- IX: Polynomial Kernel Accuracy

\begin{tabular}{ll}
\hline Dataset & Accuracy $(\%)$ \\
\hline Patient-1 & 80 \\
Patient-2 & 80 \\
Patient-3 & 70 \\
Patient-4 & 80 \\
Patient-5 & 70 \\
Patient-6 & 70 \\
Patient-7 & 70 \\
Patient-8 & 80 \\
Patient-9 & 80 \\
Patient-10 & 90 \\
Patient-11 & 80 \\
\hline
\end{tabular}

The graphical analysis of the accuracy is done at a further section of the work.

h) Quadratic Kernel Accuracy

If the number of features is far-reaching, one ought not to need to a framework to a higher dimensional space. For degree, $d=2$, the Quadratic kernel is defined as $\mathrm{K}(\mathrm{x}, \mathrm{y})=\left(\mathrm{x}^{\mathrm{T}} \mathrm{y}+\mathrm{c}\right)^{\mathrm{d}}$, where $\mathrm{x}$ and $\mathrm{y}$ are the vectors in the input space and $c$ is a free parameter. Using Quadratic Kernel method for all the patients the accuracy percentages are obtained as shown in [Table X]. That is, the nonlinear mapping does not improve the execution. Nevertheless, for the fact of confirmation, the quadratic analysis is also carried out in this work [Table X].

Table -X: Quadratic Kernel Accuracy

\begin{tabular}{ll}
\hline Dataset & Accuracy $(\%)$ \\
\hline Patient-1 & 90 \\
Patient-2 & 90 \\
Patient-3 & 70 \\
Patient-4 & 80 \\
Patient-5 & 80 \\
Patient-6 & 70 \\
Patient-7 & 80 \\
Patient-8 & 80 \\
Patient-9 & 70 \\
Patient-10 & 80 \\
Patient-11 & 80 \\
\hline
\end{tabular}

Finally, the complete accuracy analysis is visualized here [Fig 15].

Hence, it is clear to realize that the proposed framework produces a mean accuracy for modelling, detecting and classifying of $85 \%$.

Further, with the detailed understanding of the advantages of the proposed framework, this work compares this framework with parallel research outcomes. 
Accuracy Analysis

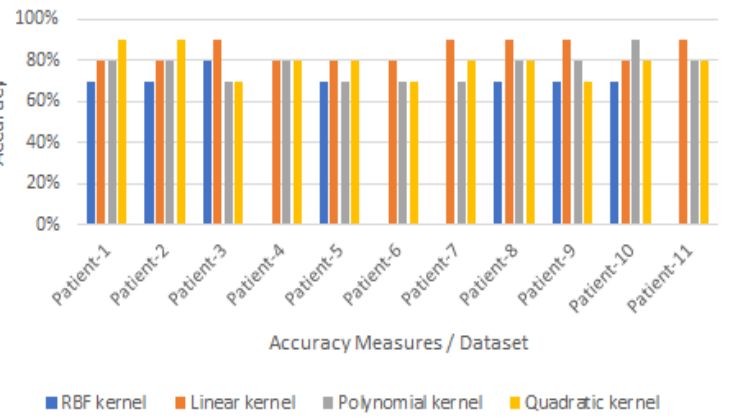

Fig. 15: Framework Accuracy Analysis.

\section{Comparative Analysis}

The obtained results from the proposed framework clearly demonstrate the improvements over the existing methods. Nonetheless, in this section of the work, in our proposed framework, Segmentation Accuracy is High, Feature Extraction is available, Classification is available, Modelling is available and Complexity is low when compared with parallel research outcomes in order to conclude a firm realization of the betterments as shown in [Table $\mathrm{XI}]$.

Table XI: Comparative Analysis

\begin{tabular}{llllll}
\hline Frameworks & Comparative Parameters & & & & Modelling \\
E. Konukoglu et al. & Moderate & Feature Extraction & Classification & Complexity \\
[23] & Not Available & Not Available & Available & High \\
Z. Wang et al. [24] & High & Not Available & Available & Available & High \\
M. A. J. Chaplain et al. & Low & Not Available & Not Available & Available & Moderate \\
[25] & Available & Available & Available & Low \\
T. Roose et al. [26] & Moderate & Not Available & Not Available & Available & High \\
C. S. Hogea et al. [28] & Low & Not Available & Available & Available & Moderate \\
J. D. Humphrey et al. & High & Available & Available & Available & Low \\
[29] & & & & \\
\hline
\end{tabular}

Thus, it is natural to realize that the proposed framework is significantly better than the other parallel research outcomes.

\section{Conclusion}

Detection, prediction or classification of the brain tumours is a long existing medical process. Nevertheless, due to the human error and higher time dependencies, it is the expected to automated the complete process. For automating any medical process, the bottleneck is the accuracy and the time complexity or the modelling complexity. Thus, a large number of parallel research outcomes are argued for acceptance. Also, the automated model or the framework is expected to be multipurpose. Hence feature and rule-based analysis is expected to be a prime component in any framework for getting the acceptance by researchers or practitioner. Hence, this work proposes a component-based framework for progressive segmentation for reducing the information loss, hybrid feature extraction for making the framework enable for other purposes, detection of tumours as primary function and classification of the tumour types for better medication. The proposed framework demonstrates a remarkable $95.45 \%$ accuracy for segmentation, astonishing $100 \%$ accuracy for feature extraction, $100 \%$ accuracy for tumour detection and $100 \%$ accuracy for class detection of the rumours. Though, the overall mean classification of the framework on BRATS dataset is $85 \%$. The major outcomes of this work are to ensure highly accurate diagnosis of tumour in brain, making features of the tumours available for any further modelling and finally, detecting the class of the tumours for early medication in order to preserve precious human life from unexpected mortalities.

\section{References}

[1] K. L. Bigos, A. R. Hariri, D. R. Weinberger, Neuroimaging Genetics: Principles and Practices, Oxford, U.K.:Oxford Univ. Press, 2016. https://doi.org/10.1093/med/9780199920211.001.0001.

[2] S. Herculano-Houzel, "The human brain in numbers: A linearly scaled-up primate brain", Frontiers Hum. Neurosci., vol. 3, pp. 31, Nov. 2009. https://doi.org/10.3389/neuro.09.031.2009.

[3] Brain Facts and Answers, Jan. 2018, [online] Available: http://www.disabled-world.com/artman/publish/brain-facts.shtml.

[4] L. M. De Angelis, "Brain tumors", New England J. Med., vol. 344, pp. 114-123, Jan. 2001 https://doi.org/10.1056/NEJM200101113440207.
[5] M. L. Goodenberger, R. B. Jenkins, "Genetics of adult glioma", Cancer Genet., vol. 205, no. 12, pp. 613-621, 2012. https://doi.org/10.1016/j.cancergen.2012.10.009.

[6] J. Meng, V. Agrahari, I. Youm, "Advances in targeted drug delivery approaches for the central nervous system tumors: The inspiration of nanobiotechnology", J. Neuroimmune Pharmacol., vol. 12, no. 1, pp. 84-98, 2016. https://doi.org/10.1007/s11481-016-9698-1.

[7] D. N. Louis et al., "The 2007 WHO classification of Tumours of the central nervous system", Acta Neuropathol., vol. 114, no. 2, pp. 97 109, 2007. https://doi.org/10.1007/s00401-007-0243-4.

[8] E. B. Claus, P. M. Black, "Survival rates and patterns of care for patients diagnosed with supratentorial low-grade gliomas", Cancer, vol. 106, no. 6, pp. 1358-1363, 2006 https://doi.org/10.1002/cncr.21733.

[9] K. A. Jaeckle et al., "Transformation of low grade glioma and correlation with outcome: An NCCTG database analysis", J. NeuroOncol., vol. 104, no. 1, pp. 253-259, 2011. https://doi.org/10.1007/s11060-010-0476-2.

[10] J. C. L. Alfonso et al., "The biology and mathematical modelling of glioma invasion: A review", J. Roy. Soc. Interface, vol. 14, no. 136, pp. 1-20, 2017. https://doi.org/10.1098/rsif.2017.0490.

[11] H. M. Byrne, T. Alarcon, M. R. Owen, S. D. Webb, P. K. Maini, "Modelling aspects of cancer dynamics: A review", Philos. Trans. Roy. Soc. London A Math. Phys. Sci., vol. 364, no. 1843, pp. 1563 1578, 2006.

[12] H. L. P. Harpold, E. C. Alvord, K. R. Swanson, "The evolution of mathematical modeling of glioma proliferation and invasion", $\mathrm{J}$. Neuropathol. Experim. Neurol., vol. 66, no. 1, pp. 1-9, 2007. https://doi.org/10.1097/nen.0b013e31802d9000.

[13] H. Hatzikirou, A. Deutsch, C. Schaller, M. Simon, K. Swanson, "Mathematical modelling of glioblastoma tumour development: A review", Math. Models Methods Appl. Sci., vol. 15, no. 11, pp. 1779-1794, 2005. https://doi.org/10.1142/S0218202505000960.

[14] A. H. Juffer, U. Marin, O. Niemitalo, J. Koivukangas, "Computer modeling of brain tumor growth", Mini Rev. Med. Chem., vol. 8, no. 14, pp. 1494-1506, 2008. https://doi.org/10.2174/138955708786786471.

[15] Y. Kam, K. A. Rejniak, A. R. A. Anderson, " Cellular modeling of cancer invasion: Integration of in silico and in vitro approaches ", J. Cellular Physiol., vol. 227, no. 2, pp. 431-438, 2012. https://doi.org/10.1002/jcp.22766.

[16] N. L. Martirosyan, E. M. Rutter, W. L. Ramey, E. J. Kostelich, Y. Kuang, M. C. Preul, "Mathematically modeling the biological properties of gliomas: A review", Math. Biosci. Eng., vol. 12, no. 4, pp. 879-905, 2015. https://doi.org/10.3934/mbe.2015.12.879.

[17] N. Meghdadi, M. Soltani, H. Niroomand-Oscuii, F. Ghalichi, "Image based modeling of tumor growth", Australas. Phys. Eng. Sci. Med., vol. 39, no. 3, pp. 601-613, 2016. https://doi.org/10.1007/s13246-016-0475-5. 
[18] A. Roniotis, K. Marias, V. Sakkalis, M. Zervakis, "Diffusive modelling of glioma evolution: A review", J. Biomed. Sci. Eng., vol. 3, pp. 501-508, May 2010. https://doi.org/10.4236/jbise.2010.35070.

[19] S. Sanga, H. B. Frieboes, X. Zheng, R. Gatenby, E. L. Bearer, V. Cristini, " Predictive oncology: A review of multidisciplinary multiscale in silico modeling linking phenotype morphology and growth ", NeuroImage, vol. 37, pp. S120-S134, May 2007. https://doi.org/10.1016/j.neuroimage.2007.05.043.

[20] Z. Wang, J. D. Butner, R. Kerketta, V. Cristini, T. S. Deisboeck, "Simulating cancer growth with multiscale agent-based modeling", Seminars Cancer Biol., vol. 30, pp. 70-78, Feb. 2015. https://doi.org/10.1016/j.semcancer.2014.04.001.

[21] L. B. Edelman, J. A. Eddy, N. D. Price, " In silico models of cancer ", Wiley Interdiscipl. Rev. Syst. Biol. Med., vol. 2, no. 4, pp. 438 459, 2010. https://doi.org/10.1002/wsbm.75.

[22] P. M. Altrock, L. L. Liu, F. Michor, "The mathematics of cancer: Integrating quantitative models", Nature Rev. Cancer, vol. 15, pp. 730-745, Nov. 2015. https://doi.org/10.1038/nrc4029.

[23] E. Konukoglu, Modeling glioma growth and personalizing growth models in medical images, Nice, France:Univ. Nice Sophia Antipolis, 2009.

[24] Z. Wang, T. S. Deisboeck, "Computational modeling of brain tumors: Discrete continuum or hybrid?" in Scientific Modeling and Simulations, Dordrecht, The Netherlands:Springer, vol. 15, pp 381-393, 2008

[25] M. A. J. Chaplain, "Avascular growth angiogenesis and vascular growth in solid tumours: The mathematical modelling of the stages of tumour development", Math. Comput. Model., vol. 23, no. 6, pp 47-87, 1996. https://doi.org/10.1016/0895-7177(96)00019-2.

[26] T. Roose, S. J. Chapman, P. K. Maini, "Mathematical models of avascular tumor growth", SIAM Rev., vol. 49, no. 2, pp. 179-208, 2007. https://doi.org/10.1137/S0036144504446291.

[27] R. P. Araujo, D. L. S. McElwain, "A history of the study of solid tumour growth: The contribution of mathematical modelling", Bull. Math. Biol., vol. 66, no. 5, pp. 1039-1091, 2004. https://doi.org/10.1016/j.bulm.2003.11.002.

[28] C. S. Hogea, B. T. Murray, J. A. Sethian, "Simulating complex tumor dynamics from avascular to vascular growth using a general level-set method", J. Math. Biol., vol. 53, no. 1, pp. 86-134, 2006. https://doi.org/10.1007/s00285-006-0378-2.

[29] J. D. Humphrey, K. R. Rajagopal, "A constrained mixture model for growth and remodeling of soft tissues", Math. Models Methods Appl. Sci., vol. 12, no. 3, pp. 407-430, 2002. https://doi.org/10.1142/S0218202502001714. 